\title{
Review of channel quality indicator estimation schemes for multi-user MIMO in 3GPP LTE/LTE-a systems
}

\begin{abstract}
Multiple-in multiple-out (MIMO) in long-term evolution (LTE) is an essential factor in achieving high speed data rates and spectral efficiency. The unexpected growth in data rate demand has pushed researchers to extend the benefits of multi-user MIMO. The multi-user MIMO system can take full advantage of channel conditions by employing efficient adjustment techniques for scheduling, and by assigning different modulation and coding rates. However, one of the critical issues affecting this feature is the appropriate estimation of channel quality indicator (CQI) to manage the allocated resources to users. Therefore, an accurate CQI estimation scheme is required for the multi-user MIMO transmission to obtain significant improvements on spectral efficiency. This paper presents overviews of multi-user MIMO in LTE/LTE-advanced systems. The link adaptation, scheduling process, and different factors that affect the reliability of CQI measurements are discussed. State-of-the-art schemes for the post-processing CQI estimation, and the comparisons of various CQI estimation schemes to support multi-user MIMO are also addressed.
\end{abstract}

Keyword: 3GPP LTE/LTE-A; Multi-user MIMO; CQI estimation; Link adaptation (LA); Scheduling 